\title{
Arterial Spin-Labeling Perfusion MRI Stratifies Progression-Free Survival and Correlates with Epidermal Growth Factor Receptor Status in Glioblastoma
}

X.J. Qiao, B.M. Ellingson, H.J. Kim, D.J.J. Wang, N. Salamon, M. Linetsky, A.R. Sepahdari, B. Jiang, J.J. Tian, S.R. Esswein, T.F. Cloughesy, A. Lai, L. Nghiemphu, and W.B. Pope

\begin{abstract}
BACKGROUND AND PURPOSE: Glioblastoma is a common primary brain tumor with a poor but variable prognosis. Our aim was to investigate the feasibility of MR perfusion imaging by using arterial spin-labeling for determining the prognosis of patients with glioblastoma.
\end{abstract}

MATERIALS AND METHODS: Pseudocontinuous arterial spin-labeling with 3D background-suppressed gradient and spin-echo was acquired before surgery on 53 patients subsequently diagnosed with glioblastoma. The calculated CBF color maps were visually evaluated by 3 independent readers blinded to patient history. Pathologic and survival data were correlated with CBF map findings. Arterial spin-labeling values in tumor tissue were also quantified by using manual fixed-size ROls.

RESULTS: Two perfusion patterns were characterized by visual evaluation of CBF maps on the basis of either the presence (pattern 1) or absence (pattern 2) of substantial hyperperfused tumor tissue. Evaluation of the perfusion patterns was highly concordant among the 3 readers $(\kappa=0.898, P<.001)$. Pattern 1 (versus pattern 2 ) was associated with significantly shorter progression-free survival by Kaplan-Meier analysis (median progression-free survival of 182 days versus 485 days, $P<.01)$ and trended with shorter overall survival $(P=.079)$. There was a significant association between pattern 1 and epidermal growth factor receptor variant III expression $(P<.01)$.

CONCLUSIONS: Qualitative evaluation of arterial spin-labeling CBF maps can be used to stratify survival and predict epidermal growth factor receptor variant III expression in patients with glioblastoma.

ABBREVIATIONS: ASL = arterial spin-labeling; EGFR = epidermal growth factor receptor; EGFRvIII = epidermal growth factor receptor variant III; GBM = glioblastoma; $\mathrm{HR}=$ hazard ratio; $\mathrm{OS}=$ overall survival; $\mathrm{PFS}=$ progression-free survival

G lioblastomas (GBMs) are the most deadly primary tumors in the CNS, with a median survival time of only 10-15 months. ${ }^{1,2}$ Increased perfusion of gliomas is associated with higher grade and shorter survival (even within a tumor grade). ${ }^{3-9}$ Information on tumor perfusion and hemodynamics can be acquired by using various MR imaging techniques. For instance, numerous studies have used dynamic susceptibility-weighted perfusion-weighted imaging, a contrast-enhanced bolus-tracking method, to determine the relative cerebral blood volume within tumors. $^{9-12}$

Received July 11, 2014; accepted after revision September 27.

From the Departments of Radiological Sciences (X.J.Q., B.M.E., H.J.K., N.S., M.L., A.R.S., J.J.T., S.R.E., W.B.P.) and Neurology (D.J.J.W., T.F.C., A.L., L.N.), David Geffen School of Medicine at University of California, Los Angeles, Los Angeles, California; and Department of Radiology (B.J.), Second Affiliated Hospital, Zhejiang University, Hangzhou, China.

Please address correspondence to Whitney B. Pope, MD, PhD, Department of Radiological Sciences, David Geffen School of Medicine, UCLA Medical Center, 10833 Le Conte Ave, BL-428/CHS, Los Angeles, CA 90095-1721; e-mail: wpope@mednet.ucla.edu

- Indicates open access to non-subscribers at www.ajnr.org

http://dx.doi.org/10.3174/ajnr.A4196
Arterial spin-labeling (ASL) is an MR imaging-based technique that measures blood flow and has the advantage, compared with DSC-PWI, of not requiring intravenous contrast injection. ASL scans of high quality can be acquired within 4.5 minutes, making it a practical approach to acquire MR perfusion imaging for clinical use. ${ }^{13,14}$ ASL has proved reliable and reproducible in the assessment of CBF in various pathologic states. Correlation has been established between DSC- and ASL-derived metrics, including relative $\mathrm{CBV}$ and $\mathrm{CBF} .{ }^{15}$ It has also been reported that ASL perfusion imaging correlates with tumor blood vessel attenuation $^{15,16}$ and is predictive of glioma grade. ${ }^{15,17,18}$ Changes in ASL-derived CBF correlate with treatment outcome for metastases following stereotactic radiosurgery. Having a completely noninvasive method, such as ASL, of determining tumor blood flow would be highly desirable, particularly if there is a correlation with survival or other outcome measures for GBM.

Tumor blood flow may also be associated with histologic markers or genetic mutations, which may impact patient prognosis and susceptibility to treatment. One such histologic marker is epidermal growth factor receptor variant III (EGFRvIII), ${ }^{19}$ the most common type of epidermal growth factor receptor (EGFR) 
variant carrying a primary oncogene effect, ${ }^{20}$ which is expressed with an overall prevalence of $20 \%-30 \%$ in GBM. ${ }^{21}$ Although the effect of EGFRvIII on patient survival has not been fully determined, ${ }^{22,23}$ detection of EGFRvIII expression is increasingly important as therapies that specifically target this receptor are being developed. Earlier studies showed that GBMs expressing EGFRvIII are resistant to chemotherapies. ${ }^{24}$ The coexpression of EGFRvIII with phosphatase and tensin homolog increases GBM sensitivity to EGFR tyrosine kinase inhibitors. ${ }^{25,26}$ The demonstrated association of DSC-PWI-derived parameters with EGFRvIII expres$\operatorname{sion}^{19}$ suggests the potential for MR perfusion imaging as a promising noninvasive marker of EGFRvIII expression.

Although ASL perfusion imaging has shown promise in the evaluation of gliomas, its role as a prognostic measure for GBM has not been fully explored, and the association between ASLderived measures and important molecular markers such as EGFRvIII is incompletely characterized. In the current study, our purpose was to determine the value of ASL perfusion imaging as a predictor of prognosis in patients with newly diagnosed GBM and as a potential marker for EGFRvIII expression.

\section{MATERIALS AND METHODS \\ Patients}

From an ongoing registry of patients diagnosed with GBM from July 2010 to July 2013 at Ronald Reagan-UCLA Medical Center, subjects were selected who had the following: 1) presurgical, prechemo-/radiation therapy MR imaging, including ASL and postcontrast T1-weighted images; 2) gross total or subtotal tumor resection followed by standard external beam radiation therapy (6000 $\pm 200 \mathrm{cGy}$, started within 3-6 weeks after surgical resection) and concomitant/adjuvant chemotherapy that included temozolomide; 3 ) a histologically confirmed diagnosis of GBM; 4) regular follow-up (once per 4-6 weeks) by a neuro-oncologist to monitor tumor recurrence either until the patient died or until March 31, 2014, when the study was terminated. Seventeen of the 53 total patients (32\%) received bevacizumab at recurrence. All patients provided informed consent to participate in our brain tumor data base containing clinical, imaging, and pathologic patient data. This study was approved by the institutional review board and was Health Insurance Portability and Accountability Act-compliant.

\section{Clinical and Histology Data}

For all selected patients, progression-free survival data were determined per the Response Assessment in Neuro-Oncology criteria. ${ }^{27}$ Progression-free survival (PFS) and overall survival (OS) were calculated from the date of surgery.

EGFRvIII expression status in postsurgical GBM tissue was collected from the patient's medical record based on standard testing procedures. ${ }^{28,29}$

\section{MR Imaging Protocols}

MR images were obtained on a 1.5T Avanto or 3T Tim Trio system (Siemens, Erlangen, Germany) by using a 12-channel head coil. The brain tumor imaging protocol included ASL, T2weighted, FLAIR, DWI, and postcontrast T1-weighted sequences. ASL scans were obtained by using a pseudocontinuous pulse se- quence with background-suppressed 3D gradient and spin-echo readout (labeling pulse duration, 1.5 seconds; postlabeling delay, 2 seconds; no flow-crushing gradient; FOV $=22 \mathrm{~cm}$; matrix size $=64 \times 64$; twenty-six 5-mm sections; generalized autocalibrating partially parallel acquisitions $=2$; TE/TR $=22 / 4000 \mathrm{~ms}$ with 30 pairs of tag and control volumes acquired within $4.5 \mathrm{~min}$ utes). An M0 image was acquired at the end of the ASL scan with a long postlabeling delay of 4 seconds and a TR of 8 seconds. ${ }^{13,14}$

\section{ASL Postprocessing and Evaluation}

ASL images were corrected for motion, pair-wise subtracted between labeled and unlabeled images and averaged to generate mean difference images and quantitative CBF maps that were calculated by using a published model. ${ }^{13}$ Motion correction was performed retrospectively by using a custom program based on principal component analysis. ${ }^{30} \mathrm{CBF}$ maps of patients with GBM $(n=$ 53) were visually evaluated by 3 independent readers (board-certified neuroradiologists with 3,7 , and 10 years of experience) blinded to patient history for focal perfusion characteristics of the tumor tissue demonstrated by conventional MR imaging, specifically postcontrast T1-weighted and T2/FLAIR sequences. Two perfusion patterns were characterized by the readers on the basis of visual evaluation of CBF maps demonstrating either the presence (pattern 1) or absence (pattern 2) of substantial and clearly recognizable areas of hyperperfused tumor tissue.

In a separate analysis, a quantitative method was applied to calculate the lesion-to-normal ratio of CBF from areas of tumor that were hyperperfused as identified by visual inspection. ROIs of $3 \times 3$ pixels in size $(10.3 \times 10.3 \mathrm{~mm}, n=3)$ were manually set in the tumor area showing the maximal perfusion $\left({ }_{\max } \mathrm{ASL}_{\text {tumor }}\right)$ by visual inspection of the CBF map, and the average value within each ROI was recorded. A second set of ROIs was drawn in the mirrored position on the contralateral hemisphere with normal brain tissue. The lesion-to-normal ratio was calculated by dividing the mean values of ${ }_{\text {max }} \mathrm{ASL}_{\text {tumor }}$ with the mean values of the ROIs in the normal region. We also compared average "hot spot" values from patterns 1 and 2 without normalization to contralateral normal brain.

\section{Statistical Analysis}

The interreader agreement in CBF map readings (pattern 1 versus pattern 2 ) from the 3 readers ( $n=53$ subjects times 3 readers) was assessed by using $\kappa$ statistics. The $\kappa$ value, also known as $\kappa$ coefficient of agreement, was calculated to evaluate the degree of consensus.

A paired $t$ test was used to compare the mean of max $\mathrm{ASL}_{\text {tumor }}$ in each subject's tumor and the mean of ASL values from ROIs in normal brain area within and between perfusion patterns (patterns 1 and 2).

ASL CBF map readings in relationship with PFS and OS were analyzed by using univariate and multivariate Cox proportional hazard models with covariates of age and sex. In cases with disagreement on CBF map readings (in which not all readers assigned the images to the same pattern), the cases were assigned to the group (hyper- versus hypoperfused) agreed on by 2 (of 3 ) readers. Ninety-five percent confidence intervals of the above covariates were calculated. The Kaplan-Meier graph was used to 

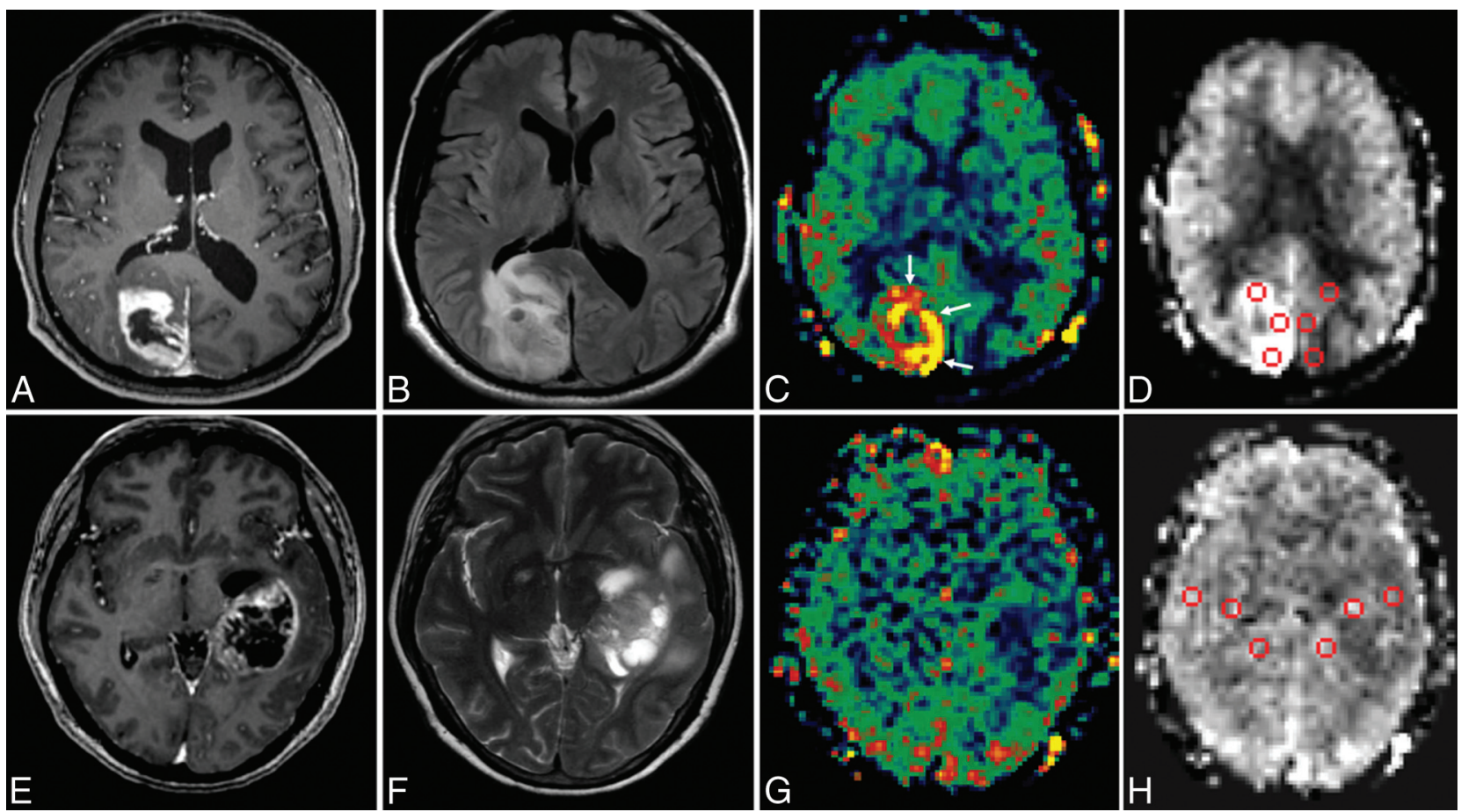

FIG 1. Qualitative and quantitative evaluation of ASL CBF maps. Conventional MR imaging (TI-weighted postcontrast imaging in $A$ and $E$, T2 FLAIR in $B$, and $T 2$ spin-echo in $F$ ) and ASL perfusion imaging $(C$ and $G$ ) were visually evaluated by 3 independent readers. Two perfusion patterns can be characterized: Pattern 1 is characteristic of the presence of substantial hyperperfused tumor tissue in the CBF map, which can be identified by the bright ring-of-fire appearance (white arrows in C); in comparison, pattern 2 corresponds to the absence of easily recognizable hyperperfused tumor tissue compatible with a relatively less perfused tumor (G). Quantitative evaluation of ASL CBF maps is performed by applying manual ROIs in the tumoral regions with a ring-of-fire appearance and the mirrored regions in the contralateral hemisphere $(D$ and $H)$.

describe ASL CBF map readings at baseline to predict PFS and OS followed by the log-rank test. The correlation between CBF map readings and EGFRvIII expression was analyzed by using the Fisher exact test.

A $P$ value of .05 was accepted as statistically significant. Statistical analysis was performed with STATA software (Version 12, 2012; StataCorp, College Station, Texas).

\section{RESULTS}

\section{Patient Characteristics}

The mean age of the 53 enrolled patients ( 28 men, 25 women) at the initial disease presentation was $60.4 \pm 13.4$ years, ranging from 29.3 to 82.4 years. By the time of the last assessment (March $31,2014), 45$ of the 53 patients $(84.9 \%)$ had tumor progression and 40 of 53 patients $(75.5 \%)$ had died.

\section{Evaluation of ASL Maps}

ASL CBF maps of patients with GBM $(n=53)$ were visually evaluated by 3 board-certified neuroradiologists blinded to patient history for focal perfusion abnormality (hyper- or hypoperfusion) in the tumor tissue. Two perfusion patterns were characterized on the basis of qualitative evaluation by the readers: Pattern 1 was characterized by the presence of substantial and easily identifiable hyperperfused tumor tissue in the CBF map, which often had a "ring-of-fire" appearance (Fig $1 A-C$ ); in comparison, pattern 2 corresponded to the absence of such easily identifiable hyperperfused tumor tissue and thus appeared in the CBF map as areas of lower perfusion in the region infiltrated by GBM (Fig $1 D-F)$. The $\kappa$ agreement test showed that evaluation of the per-
Table 1: Assessment of ASL values ${ }^{\mathrm{a}}$ in GBM by manual ROls

\begin{tabular}{|c|c|c|c|c|}
\hline & $\max _{\max } \mathrm{ASL}_{\text {tumor }} \mathrm{b}^{\mathrm{b}}$ & Control $^{\mathrm{c}}$ & $\begin{array}{l}P \text { Value } \\
\left(^{(t \text { test })^{d}}\right.\end{array}$ & $R_{\mathrm{L} / \mathrm{N}}$ \\
\hline Pattern $1(n=30)^{e}$ & $74.53 \pm 27.48$ & $35.17 \pm 10.62$ & $<.001$ & $2.27 \pm 0.99$ \\
\hline Pattern $2(n=23)^{f}$ & $32.51 \pm 22.23$ & $39.31 \pm 17.28$ & .25 & $0.81 \pm 0.37$ \\
\hline$P$ value $(t \text { test })^{d}$ & $<.001$ & .29 & - & $<.001$ \\
\hline
\end{tabular}

Note: $-\mathrm{R}_{\mathrm{L} / \mathrm{N}}$ indicates lesion-to-normal brain ratio.

${ }^{\text {a }}$ Measured in $\mathrm{mL} / 100 \mathrm{~g} / \mathrm{min}$.

${ }^{b}$ Maximal ASL values in the tumor tissue.

${ }^{c}$ ASL values taken from the contralateral hemisphere.

${ }^{\mathrm{d}} P<.05$ denotes statistical significance.

e CBF map with hyperperfused tumor tissue.

${ }^{\mathrm{f}} \mathrm{CBF}$ map with no substantial hyperperfused tumor tissue.

fusion patterns in CBF maps was highly concordant among the 3 readers $(\kappa=0.898, P<.001)$. On the basis of the most votes by readers, 30 of 53 patients (56.6\%) had CBF maps of pattern 1 , while 23 patients (43.4\%) had CBF maps of pattern 2.

To confirm that the qualitative assessment of the readers corresponded to true differences in blood flow, a quantitative analysis was performed. The quantitative analysis showed that max $\mathrm{ASL}_{\text {tumor }}$ was significantly higher than ASL values of normal brain tissue in the group with the pattern 1 CBF map, but not in the group with the pattern $2 \mathrm{CBF}$ map. When comparing groups of pattern 1 with those with pattern 2, there was a significant difference in the ${ }_{\text {max }} \mathrm{ASL}_{\text {tumor }}$ and lesion-to-normal ratio, but not in the mean values of normal brain tissue between the 2 groups (Table 1). Additionally, pattern 1 had statistically elevated CBF compared with pattern 2 , without using normalized values. Thus average CBF (in milliliters/100 grams tissue/minute) for tumor was $74.8 \pm 26.8$ for pattern 1 compared with $32.1 \pm 21.7$ for pattern $2(t$ test, $P<.001)$. 


\section{Progression-Free Survival and Overall Survival}

In a univariate Cox model, ASL CBF maps with pattern 1 (versus pattern 2) were predictive of shorter PFS (hazard ratio [HR] for progression $=2.23, P=.010)$ and trended with $\mathrm{OS}(\mathrm{HR}=1.82$, $P=.084)$. This predictive value was also significant for CBF maps with pattern 1 by using the log-rank test $(P=.008)$ (Table 2$)$.

The multivariate Cox model showed that patient age at the time of initial presentation (older than 50 years or younger than 50 years) was a significant predictor of PFS and OS ( $\mathrm{HR}=2.34$, $P=.034$; and $\mathrm{HR}=3.00, P=.024$, respectively). ASL perfusion pattern (1 versus 2) remained a significant predictor of PFS $(\mathrm{HR}=2.29, P=.009)$ and trended with $\mathrm{OS}(\mathrm{HR}=1.98, P=$ $.054)$ in the multivariate analysis (Table 3 ).

The Kaplan-Meier method with a log-rank test showed that the perfusion patterns of ASL CBF maps stratified PFS (pattern 2 versus pattern 1, a 1.66-fold increase in median PFS; $P=.008$ ) and trended with OS $(P=.079)$ (Fig 2).

Table 2: Univariate analysis: ASL CBF map of pattern 1 (versus pattern 2) is predictive of shorter progression-free survival and trended with overall survival

\begin{tabular}{ccccc}
\hline Univariate & HR (SE) & 95\% Cl & $\begin{array}{c}\text { Cox } \\
\boldsymbol{P} \text { Value }\end{array}$ & $\begin{array}{c}\text { Log-Rank } \\
\boldsymbol{P ~ V a l u e ~}^{\mathbf{a}}\end{array}$ \\
\hline PFS $(n=53)$ & $2.23(0.70)$ & $1.21-4.11$ & .010 & .008 \\
OS $(n=50)$ & $1.82(0.63)$ & $0.92-3.58$ & .084 & .079 \\
\hline
\end{tabular}

Note:-SE indicates standard error.

a $P<.05$ denotes statistical significance.

Table 3: Multivariate Cox model for PFS and OS

\begin{tabular}{|c|c|c|c|c|}
\hline Variables & HR & SE & $\begin{array}{c}P \\
\text { Value }^{a}\end{array}$ & $95 \% \mathrm{Cl}$ \\
\hline \multicolumn{5}{|c|}{ Multivariate Cox model for PFS $(n=53)$} \\
\hline ASL CBF map reading ${ }^{\mathrm{b}}$ & 2.29 & 0.72 & .009 & $1.23-4.24$ \\
\hline Patient age $(y r)^{c}$ & 2.34 & 0.94 & .034 & $1.06-5.13$ \\
\hline Sex ${ }^{d}$ & 1.41 & 0.44 & .266 & $0.77-2.59$ \\
\hline \multicolumn{5}{|c|}{ Multivariate Cox model for OS $(n=50)$} \\
\hline ASL CBF map reading & 1.98 & 0.70 & .054 & $0.99-4.00$ \\
\hline Patient age $(y r)^{c}$ & 3.00 & 1.46 & .024 & $1.16-7.80$ \\
\hline Sex ${ }^{d}$ & 1.06 & 0.36 & .870 & $0.53-2.07$ \\
\hline
\end{tabular}

Note:-SE indicates standard error.

a $P<.05$ denotes statistical significance.

${ }^{\mathrm{b}}$ By majority vote, CBF map of pattern $1(n=30)$ versus pattern $2(n=23)$.

c On initial presentation, older than 50 years $(n=42)$ versus younger than 50 years $(n=11)$.

${ }^{\mathrm{d}}$ Male $(n=28)$ versus female $(n=25)$.

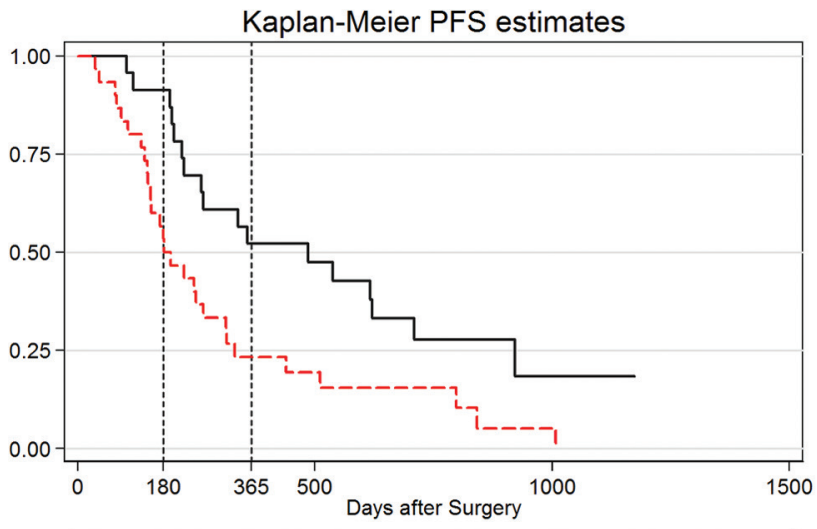

A ---- ASL high signal (Pattern 1) — ASL low signal (Pattern 2)

\section{EGFRvIII Expression}

Forty-one patients (in our patient cohort of 53, 77\%) had EGFRvIII expression examined on postsurgical GBM tissue. Among these patients, 12 were positive for EGFRvIII expression. The Fisher exact test showed a significant association between a pattern 1 CBF map and EGFRvIII expression $(P<.01)$ (Table 4$)$.

\section{DISCUSSION}

To date, most studies of GBM perfusion have focused on the role of quantitative parameters, such as relative CBV and CBF, as markers of therapy response and clinical outcome. In the present study, a qualitative examination of CBF maps was conducted in a patient cohort with newly diagnosed GBM, and the potential for predicting tumor and the correlation with EGFRvIII expression were investigated.

We observed 2 perfusion patterns: Pattern 1 was characterized by the presence of hyperperfused tumor tissue in the CBF map, which could be identified by the bright ring-of-fire appearance; in comparison, pattern 2 corresponded to the absence of hyperperfused tumor tissue and thus appeared in the CBF map as iso- or hypoperfusion in the tumoral region. These observations were supported by not only the consistency among independent readers but also quantitative analysis by using ROI-derived ASL values from both tumor and normal brain areas. The quantitative analysis confirmed the qualitative assessment that there was increased $\mathrm{CBF}$ in pattern 1 compared with pattern 2 . Conceivably, the dif-

Table 4: Association of CBF map of pattern 1 with EGFRvIII expression in $\mathrm{GBM}^{\mathrm{a}}$

\begin{tabular}{lcccc}
\hline & $\begin{array}{c}\text { Hyperperfusion } \\
(+)^{b}\end{array}$ & $\begin{array}{c}\text { Hyperperfusion } \\
(-)^{c}\end{array}$ & Total \\
\hline EGFRvIII (+) & 11 & 1 & 12 & PPV: $91.67 \%$ \\
EGFRvIII (-) & 13 & 16 & 29 & NPV: $55.17 \%$ \\
Total & 24 & 17 & 41 & \\
Sensitivity & $45.83 \%$ & & & \\
Specificity & & $94.12 \%$ & & \\
\hline
\end{tabular}

Note:-PPV indicates positive predictive value; NPV, negative predictive value; + present; -, absent.

${ }^{a}$ Of 53 patients, 41 had EGFRvIll expression examined on postsurgical GBM tissue. Fisher exact test $P$ value $=.006$.

${ }^{\mathrm{b}} \mathrm{CBF}$ map with pattern 1.

${ }^{c} \mathrm{CBF}$ map with pattern 2.

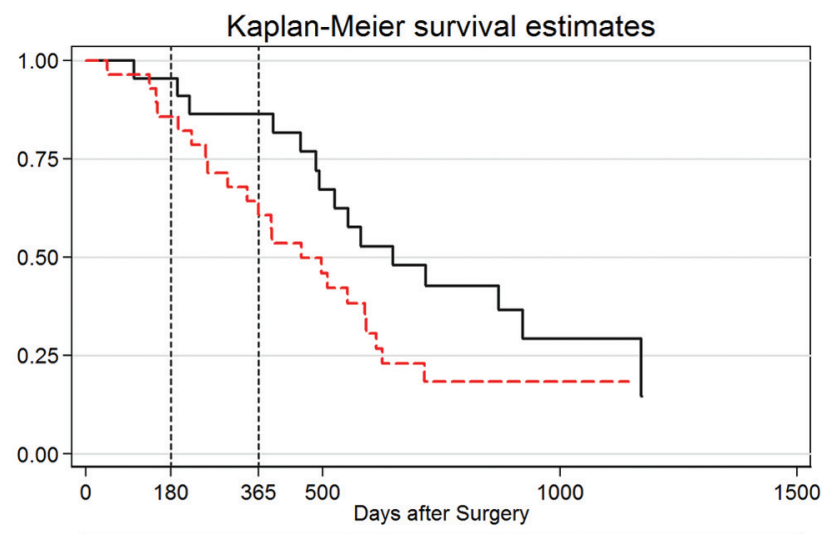

B - - - ASL high signal (Pattern 1) _ ASL low signal (Pattern 2)

FIG 2. The Kaplan-Meier curve and log-rank test for progression-free survival and overall survival. GBM perfusion patterns are examined in their relationship to PFS $(A)$ and OS (B). The $\mathrm{x}$-axis shows days after surgery (baseline) with the vertical lines noting 6- and 12-month PFS ( $A$ ) and 6- and 12 -month $\mathrm{OS}(B)$, respectively. The $y$-axis represents the percentage not progressed. 
ferentiation in the 2 perfusion patterns could be complicated in case of small tumors that are relatively difficult to identify by visual evaluation. However, this complication did not seem to cause a major problem for reader agreement because reading disagreements existed in only 4 of 53 cases (7.5\%).

The visualization of hyperperfused tumor tissue in CBF maps could prove useful for tumor biopsy. One of the clinical challenges for brain tumor biopsy has been the underestimation of the histologic grade due to the stereotactic biopsy samples being only a small portion of the tumor. Identification and sampling from the tumor section with the highest angiogenesis and the cell proliferative rate are essential for adequate evaluation of the tumor grade. Given the correlation between ASL perfusion imaging and the attenuation of blood vessels in the tumor tissue, ${ }^{15,16}$ it is reasonable to hypothesize that the macroscopic hyperperfused GBM tissue may represent a higher grade tumor. This hypothesis is supported by a study using DSC-PWI, which reported a significant association of tumor perfusion with the Ki67 index, a marker of cell proliferation. ${ }^{6}$ Therefore, qualitative evaluation of the ASL CBF map may be of added value to multiparametric MR imaging (such as MR spectroscopy, apparent diffusion coefficient, and DSC) and PET/CT as a means of identifying highly proliferative tumor tissue for biopsy.

The perfusion patterns of ASL CBF maps in relation to clinical outcomes were examined by the Kaplan-Meier curve and logrank test. ASL CBF pattern 1 (versus pattern 2) correlated with shorter PFS and trended with worse OS. Our finding is consistent with previous reports in which increased perfusion in high-grade glioma, as quantified by relative CBV derived from DSC-PWI, had significant correlation with poorer clinical outcomes. ${ }^{11,31,32}$ Quantitative analysis that correlates parameters such as volumes and mean ASL values of the hyperperfused tumor tissue with clinical outcomes could further develop the potential value of ASL CBF maps as a predictor of tumor prognosis.

EGFRvIII is a truncated extracellular mutant of EGFR protein incapable of binding the epidermal growth factor family of ligands. However, it has been shown to be a constitutively tyrosinephosphorylated persistent stimulation of growth activity. ${ }^{20}$ Tykocinski et $\mathrm{al}^{19}$ reported that relative $\mathrm{CBV}$ was a strong quantitative indicator of EGFRvIII expression in a study using DSC-PWI. Our study demonstrates a correlation between ASL CBF pattern 1 and EGFRvIII expression with a low sensitivity but high specificity. One possible explanation for the observed low sensitivity is that the hyperperfused tissue in GBM could be caused by effects from multiple genes and/or cross-talk between multiple cell-signaling pathways. For instance, alternative pathways are suggested by reports showing upregulation of the vascular endothelial growth factor with increased relative CBV. ${ }^{19}$ The high specificity, however, might indicate that EGFRvIII expression alone has enough impact to generate the phenotype of macroscopic hyperperfused tissue in GBM.

The limitations of the present study include variability in therapies of the patient cohort, which may independently affect clinical outcome. However, all patients received standard therapy with radiation treatment and temozolomide, and recent data suggest that bevacizumab (which some patients received), while potentially prolonging progression-free survival by several months, has little impact on survival time. ${ }^{33}$ Thus, the overall impact of therapy variability may be limited. Our study was also limited by a small sample size, reducing our ability to detect a potentially significant difference in overall survival (not merely progressionfree survival) between patient cohorts. The potential value of ASL classifiers as a predictor of tumor prognosis and a marker of EGFRvIII expression requires verification in prospective studies. Although 2 perfusion patterns were identified in the CBF maps, a finding supported by data from quantitative analysis, potentially a more comprehensive quantitative approach, such as histogram analysis, could yield further classifiers of prognostic or predictive significance. However, our qualitative methodology has the advantage of not being reliant on extensive postprocessing, suggesting it could find utility in the real-time work flow of a busy clinical practice setting. One of the challenges for the widespread translation of ASL in clinical practice is that there is a wide range of ASL techniques and imaging protocols available from different vendors and developers. To address this issue, the recent ASL white paper $^{34}$ recommended pseudocontinuous ASL with a 3D readout and a relatively long postlabeling delay (eg, 2 seconds), which was used in the current study.

\section{CONCLUSIONS}

The qualitative evaluation of ASL CBF maps provides an expeditious method with high interobserver reliability that stratifies survival and predicts EGFRvIII expression in patients with GBM.

Disclosures: Benjamin M. Ellingson—RELATED: Grant: Genentech/Roche, Siemens. Danny J.J. Wang-RELATED: Grant: National Institutes of Health, ${ }^{*}$ Comments: R01 EB014922/EB/NIBIB, R01 MH080892/MH/NIMH, R01 NS081077/NS/NINDS. Albert Lai-UNRELATED: Consultancy: Genentech/Roche, ${ }^{\star}$ Comments: Avastin Scientific Advisory Board. Timothy F. Cloughesy-UNRELATED: Consultancy: VBL, Proximagen, Tocagen, Genentech/Roche, Celgene, Lpath; Expert Testimony: Roche. *Money paid to the institution.

\section{REFERENCES}

1. Stupp R, Mason WP, van den Bent MJ, et al. Radiotherapy plus concomitant and adjuvant temozolomide for glioblastoma. $N \mathrm{Engl}$ J Med 2005;352:987-96

2. Van Meir EG, Hadjipanayis CG, Norden AD, et al. Exciting new advances in neuro-oncology: the avenue to a cure for malignant glioma. CA Cancer J Clin 2010;60:166-93

3. Louis DN, Ohgaki H, Wiestler OD, et al. The 2007 WHO classification of tumours of the central nervous system. Acta Neuropathol 2007;114:97-109

4. Arvinda HR, Kesavadas C, Sarma PS, et al. Glioma grading: sensitivity, specificity, positive and negative predictive values of diffusion and perfusion imaging. J Neurooncol 2009;94:87-96

5. Law M, Yang S, Wang H, et al. Glioma grading: sensitivity, specificity, and predictive values of perfusion MR imaging and proton MR spectroscopic imaging compared with conventional MR imaging. AJNR Am J Neuroradiol 2003;24:1989-98

6. Alexiou GA, Zikou A, Tsiouris S, et al. Correlation of diffusion tensor, dynamic susceptibility contrast MRI and (99m)Tc-tetrofosmin brain SPECT with tumour grade and Ki-67 immunohistochemistry in glioma. Clin Neurol Neurosurg 2014;116:41-45

7. Yoon JH, Kim JH, Kang WJ, et al. Grading of cerebral glioma with multiparametric MR imaging and 18F-FDG-PET: concordance and accuracy. Eur Radiol 2014;24:380 - 89

8. Weber MA, Henze M, Tüttenberg J, et al. Biopsy targeting gliomas: do functional imaging techniques identify similar target areas? Invest Radiol 2010;45:755-68

9. Hu LS, Eschbacher JM, Dueck AC, et al. Correlations between per- 
fusion MR imaging cerebral blood volume, microvessel quantification, and clinical outcome using stereotactic analysis in recurrent high-grade glioma. AJNR Am J Neuroradiol 2012;33:69-76

10. LaViolette PS, Cohen AD, Prah MA, et al. Vascular change measured with independent component analysis of dynamic susceptibility contrast MRI predicts bevacizumab response in high-grade glioma. Neuro Oncol 2013;15:442-50

11. Mangla R, Ginat DT, Kamalian S, et al. Correlation between progression free survival and dynamic susceptibility contrast MRI perfusion in WHO grade III glioma subtypes. J Neurooncol 2014;116: 325-31

12. Law M, Young RJ, Babb JS, et al. Gliomas: predicting time to progression or survival with cerebral blood volume measurements at dynamic susceptibility-weighted contrast-enhanced perfusion MR imaging. Radiology 2008;247:490-98

13. Wang DJ, Alger JR, Qiao J, et al. The value of arterial spin-labeled perfusion imaging in acute ischemic stroke: comparison with dynamic susceptibility contrast-enhanced MRI. Stroke 2012;43: $1018-24$

14. Wang DJ, Alger JR, Qiao J, et al. Multi-delay multi-parametric arterial spin-labeled perfusion MRI in acute ischemic stroke-comparison with dynamic susceptibility contrast enhanced perfusion imaging. Neuroimage Clin 2013;3:1-7

15. Warmuth C, Gunther M, Zimmer C. Quantification of blood flow in brain tumors: comparison of arterial spin labeling and dynamic susceptibility weighted contrast-enhanced MR imaging. Radiology 2003;228:523-32

16. Noguchi T, Yoshiura T, Hiwatashi A, et al. Perfusion imaging of brain tumors using arterial spin-labeling: correlation with histopathologic vascular density. AJNR Am J Neuroradiol 2008;29:688-93

17. Chawla S, Wang S, Wolf RL, et al. Arterial spin-labeling and MR spectroscopy in the differentiation of gliomas. AJNR Am J Neuroradiol 2007;28:1683-89

18. Kim HS, Kim SY. A prospective study on the added value of pulsed arterial spin-labeling and apparent diffusion coefficients in the grading of gliomas. AJNR Am J Neuroradiol 2007;28:1693-99

19. Tykocinski ES, Grant RA, Kapoor GS, et al. Use of magnetic perfusion-weighted imaging to determine epidermal growth factor receptor variant III expression in glioblastoma. Neuro Oncol 2012; 14:613-23

20. Zhan Y, O'Rourke DM. SHP-2-dependent mitogen-activated protein kinase activation regulates EGFRvIII but not wild-type epidermal growth factor receptor phosphorylation and glioblastoma cell survival. Cancer Res 2004;64:8292-98
21. Gan HK, Kaye AH, Luwor RB. The EGFRvIII variant in glioblastoma multiforme. J Clin Neurosci 2009;16:748-54

22. Feldkamp MM, Lala P, Lau N, et al. Expression of activated epidermal growth factor receptors, Ras-guanosine triphosphate, and mitogen-activated protein kinase in human glioblastoma multiforme specimens. Neurosurgery 1999;45:1442-53

23. Pelloski CE, Ballman KV, Furth AF, et al. Epidermal growth factor receptor variant III status defines clinically distinct subtypes of glioblastoma. J Clin Oncol 2007;25:2288-94

24. Nagane M, Levitzki A, Gazit A, et al. Drug resistance of human glioblastoma cells conferred by a tumor-specific mutant epidermal growth factor receptor through modulation of Bcl-XL and caspase3-like proteases. Proc Natl Acad Sci U S A 1998;95:5724-29

25. Mellinghoff IK, Wang MY, Vivanco I, et al. Molecular determinants of the response of glioblastomas to EGFR kinase inhibitors. $N$ Engl J Med 2005;353:2012-24

26. Raizer JJ. HER1/EGFR tyrosine kinase inhibitors for the treatment of glioblastoma multiforme. J Neurooncol 2005;74:77-86

27. Wen PY, Macdonald DR, Reardon DA, et al. Updated response assessment criteria for high-grade gliomas: response assessment in neuro-oncology working group. J Clin Oncol 2010;28:1963-72

28. Louis DN, Holland EC, Cairncross JG. Glioma classification: a molecular reappraisal. Am J Pathol 2001;159:779-86

29. Yoshimoto K, Dang J, Zhu S, et al. Development of a real-time RTPCR assay for detecting EGFRvIII in glioblastoma samples. Clin Cancer Res 2008; 14:488-93

30. Wang J, Alsop DC, Song HK, et al. Arterial transit time imaging with flow encoding arterial spin tagging (FEAST). Magn Reson Med 2003;50:599-607

31. Schmainda KM, Prah M, Connelly J, et al. Dynamic-susceptibility contrast agent MRI measures of relative cerebral blood volume predict response to bevacizumab in recurrent high-grade glioma. Neuro Oncol 2014;16:880-88

32. Essock-Burns E, Lupo JM, Cha S, et al. Assessment of perfusion MRI-derived parameters in evaluating and predicting response to antiangiogenic therapy in patients with newly diagnosed glioblastoma. Neuro Oncol 2011;13:119-31

33. Piccioni DE, Lai A. Deferred use of bevacizumab for recurrent glioblastoma is not associated with diminished efficacy. Neuro Oncol 2014;16:1427-28

34. Alsop DC, Detre JA, Golay X, et al. Recommended implementation of arterial spin-labeled perfusion MRI for clinical applications: a consensus of the ISMRM perfusion study group and the European consortium for ASL in dementia. Magn Reson Med 2014 Apr 8. [Epub ahead of print] 